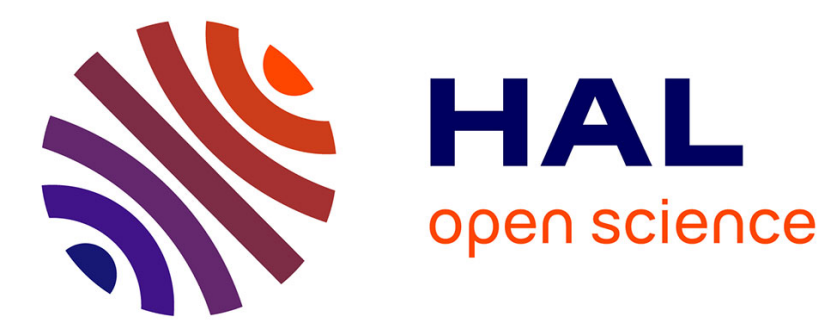

\title{
Finite dimensional global attractor for a semi-discrete fractional nonlinear Schrödinger equation
}

Caterina Calgaro, Olivier Goubet, Ezzeddine Zahrouni

\section{To cite this version:}

Caterina Calgaro, Olivier Goubet, Ezzeddine Zahrouni. Finite dimensional global attractor for a semidiscrete fractional nonlinear Schrödinger equation. Mathematical Methods in the Applied Sciences, 2017, 10.1002/mma.4409 . hal-01388788

\section{HAL Id: hal-01388788 \\ https://hal.science/hal-01388788}

Submitted on 28 Oct 2016

HAL is a multi-disciplinary open access archive for the deposit and dissemination of scientific research documents, whether they are published or not. The documents may come from teaching and research institutions in France or abroad, or from public or private research centers.
L'archive ouverte pluridisciplinaire HAL, est destinée au dépôt et à la diffusion de documents scientifiques de niveau recherche, publiés ou non, émanant des établissements d'enseignement et de recherche français ou étrangers, des laboratoires publics ou privés. 


\title{
Finite dimensional global attractor for a semi-discrete fractional nonlinear Schrödinger equation
}

\author{
Caterina Calgaro
}

Univ. Lille, CNRS, UMR 8524 - Laboratoire Paul Painlevé, F-59000 Lille, France.

caterina.calgaro@math.univ-lille1.fr

Olivier Goubet

LAMFA UMR 7352 CNRS UPJV, 33 rue saint-Leu, 80039 Amiens Cedex, France.

olivier.goubet@u-picardie.fr

EZZEDDINE ZAHROUNI

FSEG Nabeul, University of Carthage,8000 Nabeul, Tunisie.

ezzeddine.zahrouni@fsm.rnu.tn

\begin{abstract}
We consider a semi-discrete in time Crank-Nicolson scheme to discretise a weakly damped forced nonlinear fractional Schrödinger equation $u_{t}-$ $i(-\Delta)^{\alpha} u+i|u|^{2} u+\gamma u=f$ for $\alpha \in\left(\frac{1}{2}, 1\right)$ considered in the the whole space $\mathbb{R}$. We prove that such semi-discrete equation provides a discrete infinite dimensional dynamical in $H^{\alpha}(\mathbb{R})$ that possesses a global attractor in $H^{\alpha}(\mathbb{R})$. We show also that if the external force is in a suitable weighted Lebesgue space then this global attractor has a finite fractal dimension.
\end{abstract}

AMS: 35Q55, 35B41, 37L30.

keywords: Nonlinear Schrödinger equations, Crank-Nicolson scheme, global attractor, fractal dimension. 


\section{Introduction}

The fractional nonlinear Schrödinger in the whole line $\mathbb{R}$ reads

$$
u_{t}-i(-\Delta)^{\alpha} u+i|u|^{2} u=0,
$$

where the unknown $u=u(t, x)$ is a complex valued function defined on $\mathbb{R} \times \mathbb{R}$. It was derived by Laskin $[18,19]$ as a result of expanding the Feynman path integral, substituting to the usual Brownian path the so-called Levy quantum mechanical paths. This is a fundamental equation of fractional quantum mechanics, where Levy path provide fractional Laplace operators instead of the usual Laplace operator. For the mathematical point of vue, the initial value problem was addressed recently by numerous authors. The well-posedness of $(1)$ for $\alpha \in\left(\frac{1}{2}, 1\right)$ in $L^{2}(\mathbb{R})$ was established by B. Guo and Z. Huo [15]. A general theory for well-posedness in Sobolev spaces for fractional NLS equations in $\mathbb{R}^{N}$ is given by Y. Hong and Y. Sire in [17].

The equation (1) is a conservative equation. In some physical contexts, an external forcing term and some damping effects have to be taken into account. This leads to the following weakly damped fractional nonlinear Schrödinger equation

$$
u_{t}-i(-\Delta)^{\alpha} u+i|u|^{2} u+\gamma u=f,
$$

where $\gamma>0$ denotes the damping parameter and $f$ is the external forcing term. We assume that the source term $f$ is independent of time and that $f$ belongs to $L^{2}(\mathbb{R})$.

We supplement (2) with the initial condition

$$
u(0, .)=u_{0} \in H^{\alpha}(\mathbb{R}),
$$

where $H^{\alpha}(\mathbb{R})$ is the fractional Sobolev space associated to the equation (see the very definition of $H^{\alpha}(\mathbb{R})$ below).

This equation provides an infinite-dimensional dynamical system, in the frame-

work described in [25], [16], [22] and [21]. Results concerning the existence of a global attractor in $H^{\alpha}$ for this fractional equation appear in [6]. Equation (2) is the fractional analog of the usual weakly damped cubic nonlinear Schrödinger equation that was first studied in [20] and [28]. Further issues as the regularity of global attractors for these NLS equations were addressed in [10], [2] in the one dimensional case, and in [11], [12] for the 2D case. 
In this article, we address the discrete counterpart of these continuous dynamical systems. Actually, we consider a suitable semi-discrete fractional nonlinear Schrödinger equation introducing a suitable Crank-Nicolson scheme in time, keeping the space variable continuous. These numerical schemes first introduced in [8] (see also [23]) for numerical purposes, provide also discrete infinite-dimensional dynamical systems. This point of view for classical NLS equations appear in [14]. We do not address here alternate discretizations for NLS as respectively splitting methods (see [4] and the references therein) or respectively relaxation methods introduced in [3].

Fruitful results about Crank-Nicolson scheme appear in the infinite dimensional dynamical system literature: for the case when the the space variable $x$ belongs to a finite interval, with periodic boundary conditions, it was proved in [14] the existence of a global attractor. It turns out that this result was also obtained in the case where the variable space $x$ belongs to $\mathbb{T}^{2}$ in [9]. It is worth recalling that the discrete Crank-Nicolson scheme in both space and time variable for (2) was studied in [27] and [1] for weakly damped Nonlinear Schrödinger Equation. The discrete in time relaxation scheme for a non local nonlinear Schrödinger equation was studied in [13]. The discrete dynamical system provided by the Crank-Nicolson scheme to (2) is new to our knowledge.

The rest of the article is organized as follows. Section 2 is devoted to the derivation of the scheme and the main results. Section 3 deals the mathematical framework, where we state and prove a nonstandard commutator estimate. Section 4 , we focus on the existence and uniqueness of the discrete solution. Finally, in section 5 we prove the existence of a compact global attractor in the phase space $H^{\alpha}$. We then prove, assuming moreover that the external force has some decay at the infinity, that this global attractor has a finite fractal dimension.

\section{Derivation of the scheme and main results}

\subsection{The Crank-Nicolson Scheme}

In this section, we introduce a Crank-Nicolson scheme associated to (2), following an idea of M. Abounouh. Equation (2) has a unique solution $u \in C\left([0, \infty), H^{\alpha}\right) \cap$ $C^{1}\left([0, \infty), H^{-\alpha}\right)$ (see [17]). Alternatively, the equation reads 


$$
\frac{\partial}{\partial t}\left(e^{\gamma t} u(t)\right)-i(-\Delta)^{\alpha}\left(e^{\gamma t} u(t)\right)+i|u(t)|^{2}\left(e^{\gamma t} u(t)\right)=e^{\gamma t} f
$$

where $\Delta u=\partial_{x x} u$.

For a given time step $\tau>0$, we consider the uniform time sequence $\left(t^{n}\right)_{n}$ defined by $t^{n}=n \tau$ and we integrate (4) in time on the interval $\left[t^{n}, t^{n+1}\right]$ to get

$$
\begin{array}{r}
e^{\gamma t^{n+1}} u\left(t^{n+1}\right)-e^{\gamma t^{n}} u\left(t^{n}\right)+ \\
\int_{t^{n}}^{t^{n+1}}\left[-i(-\Delta)^{\alpha} e^{\gamma t} u(t)+i|u(t)|^{2} e^{\gamma t} u(t)\right] d t=\frac{e^{\gamma t^{n+1}}-e^{\gamma t^{n}}}{\gamma} f .
\end{array}
$$

Set $\left(u^{n}\right)_{n}$ to be such that $u^{n} \sim u\left(t^{n}\right)$, where $u$ is a solution to (2). Using the the trapezoidal rule to approximate the time integral

$$
\int_{t^{n}}^{t^{n+1}} g(s) d s \sim \frac{\tau}{2}\left(g\left(t^{n+1}\right)+g\left(t^{n}\right)\right),
$$

and the approximation of the nonlinearity in [8] (that goes back to [24], according to the reference [23]), we are led to

$$
\begin{array}{r}
\frac{1}{\tau}\left(u^{n+1}-\delta u^{n}\right)-\frac{i}{2}(-\Delta)^{\alpha}\left(u^{n+1}+\delta u^{n}\right)+\frac{i}{4}\left(\left|u^{n+1}\right|^{2}+\left|u^{n}\right|^{2}\right)\left(u^{n+1}+\delta u^{n}\right)= \\
f \simeq \frac{1-\delta}{\gamma \tau} f,
\end{array}
$$

where $\delta=e^{-\gamma \tau}$, and $\gamma, \tau>0, f \in L^{2}(\mathbb{R}), u^{0} \in H^{\alpha}$. We have used in (5) that $f$ is time independent function and the approximation $\frac{1-\delta}{\gamma \tau} \simeq 1$ that is valid if $\tau$ is small enough.

\subsection{Main results}

Our first main result is the following. The result is concerned with the wellposedness of the scheme.

Theorem 1 For any $u^{n}$ in $H^{\alpha}$, there exists at least one solution $u^{n+1}$ of (7). Let $S: H^{\alpha} \longrightarrow H^{\alpha}, u^{n} \mapsto u^{n+1}$ the multivalued function defined by (7). There exists a bounded set $\mathcal{E} \subset H^{\alpha}$ such that $S(\mathcal{E}) \subset \mathcal{E}$, and such that for $\tau>0$ small enough, the map $S$ is continuous and one-to-one on $\mathcal{E}$.

Our second result is concerned with the existence of the global attractor for the $\operatorname{map} S: \mathcal{E} \rightarrow \mathcal{E}$. 
Theorem 2 The discrete dynamical system $\left(S^{n}\right)_{n \in \mathbb{N}}$ defined on the set $\mathcal{E}$ by $S u^{n}=u^{n+1}$ possesses a compact global attractor $\mathcal{A}_{\tau}$ in $H^{\alpha}$.

Our last result is concerned with the fractal dimension of the attractor, assuming that the external force $f$ has some decay at the infinity.

Theorem 3 Assume moreover that the external force $f$ belongs to $L^{2}(\mathbb{R},(1+$ $\left.\left.x^{2}\right) d x\right)$, i.e $\int|f(x)|^{2}\left(1+x^{2}\right) d x<+\infty$. Then $\mathcal{A}_{\tau}$ is included in $L^{2}\left(\mathbb{R},\left(1+x^{2}\right) d x\right)$ and has finite fractal dimension in $H^{\alpha} \cap L^{2}\left(\mathbb{R},\left(1+x^{2}\right) d x\right)$.

Remark 1 The assumption that the datum $f$ belongs to some weighted space in order to ensure that the global attractor has finite dimension is natural, since it is still an open question to remove this assumption for classical NLS equation.

\section{Mathematical framework and notations}

\section{$3.1 \quad$ Notations}

The Fourier transform of $u$ is denoted by either $\hat{u}$ or $\mathcal{F}(u)$ and defined by

$$
\mathcal{F}(u)(\xi)=\int_{\mathbb{R}} u(x) e^{-i x \xi} d x .
$$

We consider the homogeneous fractional pseudo-differential operator $\Lambda^{s}$ defined by

$$
\Lambda^{s}(u)(x)=\mathcal{F}^{-1}\left(|\xi|^{s} \hat{u}(\xi)\right)(x) .
$$

We recall some functional spaces that we will use in the sequel.

For $1 \leq r \leq \infty, s \in \mathbb{R}$, the spaces $L^{r}$ and $H^{s}$ will denote the usual Lebesgue $L^{r}(\mathbb{R})$ and Sobolev spaces $H^{s}(\mathbb{R})$ of complex valued functions. The space $\mathcal{S}$ denotes the Schwartz class. The spaces $L^{2}$ and $H^{s}$ are Hilbert spaces endowed respectively with the following inner product,

$$
(u, v)_{L^{2}}=\operatorname{Re} \int_{\mathbb{R}} u \bar{v} d x,
$$

and

$$
2 \pi((u, v))_{H^{s}}=\operatorname{Re} \int_{\mathbb{R}}\left(1+|\xi|^{2}\right)^{s} \hat{u}(\xi) \overline{\hat{v}}(\xi) d \xi .
$$

The norms on these space will be denoted simply by $\|.\|_{H^{s}}$ for instance. Throughout this article $C$ and $K$ denote numerical constants that may vary from one line to one another, $K$ depending on the data $\gamma,\|f\|_{L^{2}}$. 


\subsection{Useful results}

We recall here results from functional analysis that will be used in the sequel. The first one is related to the Brouwer fixed point theorem.

Lemma 1 ([5] and [26]) Let $X$ a finite dimensional space endowed with a scalar product [.,.] and consider a continuous mapping $F: X \rightarrow X$. Suppose that there exists $R_{0}>0$, such that for all $w$ satisfying $[w, w]^{\frac{1}{2}}=R_{0}$ we have $[F(w), w]>0$. Then there exists $w^{*},\left[w^{*}, w^{*}\right]^{\frac{1}{2}}<R_{0}$ such that $F\left(w^{*}\right)=0$.

For $\alpha>\frac{1}{2}$, we recall that $H^{\alpha}$ is an algebra and that it is included in $L^{\infty}$. Moreover, we have the interpolation inequality

$$
\|u\|_{L^{\infty}} \leq C_{\alpha}\|u\|_{L^{2}}^{1-\frac{1}{2 \alpha}}\left\|\Lambda^{\alpha} u\right\|_{L^{2}}^{\frac{1}{2 \alpha}}
$$

To check (10), we proceed as follows. Thanks to the inverse Fourier transform we just have to provide an upper bound to the $L^{1}$ norm of $\hat{u}$. Consider $R>0$. We have, thanks to Cauchy-Schwarz inequality

$$
\|\hat{u}\|_{L^{1}} \leq \int_{|\xi| \leq R}|\hat{u}|+\int_{|\xi| \geq R} \frac{|\xi|^{\alpha}}{|\xi|^{\alpha}}|\hat{u}| \leq c_{\alpha}\left(\sqrt{R}\|u\|_{L^{2}}+R^{\frac{1}{2}-\alpha}\left\|\Lambda^{\alpha} u\right\|_{L^{2}}\right)
$$

a suitable choice of $R$ yields (10). It is worth to point out that we may substitute $\|u\|_{H^{\alpha}}$ to $\left\|\Lambda^{\alpha} u\right\|_{L^{2}}$ in the right hand side of (10).

We finally state and prove the following commutator estimate.

Proposition 1 Consider $\varphi \in \mathcal{S}$ and $u \in H^{\alpha}$. Then

$$
\left\|\left[(-\Delta)^{\alpha}, \varphi\right] u\right\|_{2} \leq C_{\alpha}\left[\|\xi \hat{\varphi}\|_{L^{1}}\|u\|_{H^{2 \alpha-1}}+\left\||\xi|^{2 \alpha} \hat{\varphi}\right\|_{L^{1}}\|u\|_{2}\right]
$$

Remark 2 Let us recall that $2 \alpha-1<\alpha$. We then for instance can bound by above $\|u\|_{H^{2 \alpha-1}}$ by $\|u\|_{H^{\alpha}}$ or any norm $H^{\beta}$ for $\beta \in(2 \alpha-1, \alpha]$.

Proof. We note that

$$
\mathcal{F}(u v)(\xi)=\int_{\mathbb{R}} \hat{u}(\eta) \hat{v}(\xi-\eta) d \eta
$$

so

$$
\mathcal{F}(-\Delta)^{\alpha}(\varphi u)(\xi)=|\xi|^{2 \alpha} \int_{\mathbb{R}} \hat{\varphi}(\eta) \hat{u}(\xi-\eta) d \eta
$$


and similarly,

$$
\mathcal{F}\left(\varphi(-\Delta)^{\alpha} u\right)(\xi)=\int_{\mathbb{R}} \hat{\varphi}(\eta)|\xi-\eta|^{2 \alpha} \hat{u}(\xi-\eta) d \eta
$$

Hence for the commutator $\left[(-\Delta)^{\alpha}, \varphi\right]$ we have,

$$
\mathcal{F}\left(\left[(-\Delta)^{\alpha}, \varphi\right] u\right)(\xi)=\int_{\mathbb{R}}\left(|\xi|^{2 \alpha}-|\xi-\eta|^{2 \alpha}\right) \hat{\varphi}(\eta) \hat{u}(\xi-\eta) d \eta
$$

We now use, recalling that $2 \alpha-1 \in(0,1)$,

$$
\left.|| \xi\right|^{2 \alpha}-|\xi-\eta|^{2 \alpha}\left|\leq \tilde{c}_{\alpha} \max \left(|\xi|^{2 \alpha-1},|\xi-\eta|^{2 \alpha-1}\right)\right| \eta \mid \leq c_{\alpha}\left(|\xi|^{2 \alpha-1}|\eta|+|\eta|^{2 \alpha}\right)
$$

using that $|\xi|^{2 \alpha-1} \leq c_{\alpha}^{\prime}\left(|\xi-\eta|^{2 \alpha-1}+|\eta|^{2 \alpha-1}\right)$. Hence we bound by above the integral in (16) by two integrals, accordingly to the upper bound (17). The square of the first integral is bounded as follows

$$
\begin{array}{r}
\left|\int_{\mathbb{R}}\right| \eta|| \hat{\varphi}(\eta)|| \xi-\left.\left.\eta\right|^{2 \alpha-1}|\hat{u}(\xi-\eta)| d \eta\right|^{2} \\
\leq\left(\int_{\mathbb{R}}|\eta||\hat{\varphi}(\eta)| d \eta\right)\left(\int_{\mathbb{R}}|\eta||\hat{\varphi}(\eta)||\xi-\eta|^{4 \alpha-2}|\hat{u}(\xi-\eta)|^{2} d \eta\right) .
\end{array}
$$

Integrating in $\xi$ leads to an upper bound in $c|| u\left\|_{H^{2 \alpha-1}}\right\| \xi \hat{\varphi} \|_{L^{1}}$ due to Plancherel formula. We then have the first term in the right hand side of (12). The second integral reads $\left.\left|\int_{\mathbb{R}}\right| \hat{\varphi}(\eta)|| \eta\right|^{2 a}|\hat{u}(\xi-\eta)| d \eta \mid$, and we proceed exactly as above. This completes the proof of the lemma.

We complete this section by the discrete Gronwall lemma that will be of constant use throughout this article.

Lemma 2 Set $\delta<1$. Consider a sequence $a^{n}$ such that $a^{n+1} \leq \delta a^{n}+c$. Then $a^{n} \leq \delta^{n} a^{0}+\frac{c}{1-\delta}$. 


\section{Proof of Theorem 1}

In this section, we prove that the Crank-Nicolson scheme (7) is well-posed and provides the existence of a discrete dynamical system $S: u^{n} \rightarrow u^{n+1}$. We follow here the method in [9]. We first prove that for a given $u^{n}$ there exists at least a $u^{n+1}$ that solves the equation (7). The existence results relies on some compactness argument. The drawback of the method is that the uniqueness is not ensured. We then prove that there exists some absorbing set that is invariant by $S$. We eventually prove that restricted to this absorbing set, and that for $\tau$ small enough, $S$ is a continuous one-to-one map.

\subsection{A Galerkin Method}

Consider here $\left(\varphi_{k}\right)_{k \in \mathbb{N}^{*}}$ a basis of $H^{\alpha}$. We assume that this family is orthogonal in $L^{2}$. Set $V_{N}=\operatorname{Span}\left\{\varphi_{1}, \cdots, \varphi_{N}\right\}$ the finite dimensional subspace, and introduce $P_{N}: H^{\alpha} \rightarrow V_{N}$ the $L^{2}$-orthogonal projector.

We seek $w^{N}$ in $V_{N}$ that is solution to a Galerkin approximation of (7) that reads as, for any $\varphi^{N} \in V_{N}$,

$$
\begin{array}{r}
\left(\frac{w^{N}-\delta P_{N} u^{n}}{\tau}-i \Lambda^{2 \alpha} \frac{\left(w^{N}+\delta P_{N} u^{n}\right)}{2}+i\left(\left|w^{N}\right|^{2}+\left|u^{n}\right|^{2}\right) \frac{\left(w^{N}+\delta P_{N} u^{n}\right)}{4}, \varphi^{N}\right)_{L^{2}} \\
=\left(P_{N} f, \varphi^{N}\right)_{L^{2}}
\end{array}
$$

We now state and prove

Proposition 2 The equation (19) has at least a solution $w^{N}$ that satisfies moreover

$$
\left\|w^{N}\right\|_{L^{2}} \leq 4 \delta\left\|u^{n}\right\|_{L^{2}}+\tau\|f\|_{L^{2}} .
$$

Proof : Consider $P_{N}^{*}: H^{-\alpha} \rightarrow V_{N}$ defined as $\left(P_{N}^{*} \mu, \phi\right)_{L^{2}}=<\mu, P_{N} \phi>_{H^{-\alpha}, H^{\alpha}}$, for any $\phi \in L^{2}$. Introduce, setting $w=w^{N}+\delta P_{N} u^{n}$,

$$
F(w)=P_{N}^{*}\left(\frac{w-2 \delta P_{N} u^{n}}{\tau}-i \Lambda^{2 \alpha} \frac{w}{2}+i\left(\left|w-\delta P_{N} u^{n}\right|^{2}+\left|u^{n}\right|^{2}\right) \frac{w}{4}-P_{N} f\right) .
$$


This defines a continuous map $F: V_{N} \rightarrow V_{N}$ such that $w^{N}$ solves (19) if and only if $F\left(w^{N}+\delta P_{N} u^{n}\right)=0$. Let us observe that

$$
(F(w), w)_{L^{2}}=\frac{1}{\tau}\left(\left\|w^{N}\right\|_{L^{2}}^{2}-\delta^{2}\left\|P_{N} u^{n}\right\|_{L^{2}}^{2}\right)-\left(P_{N} f, w^{N}+\delta P_{N} u^{n}\right) .
$$

Therefore

$$
(F(w), w)_{L^{2}} \geq\left(\left\|w^{N}\right\|_{L^{2}}+\delta\left\|P_{N} u^{n}\right\|_{L^{2}}\right)\left(\frac{\left\|w^{N}\right\|_{L^{2}}-\delta\left\|P_{N} u^{n}\right\|_{L^{2}}}{\tau}-\|f\|_{L^{2}}\right) .
$$

Set $R_{0}=3 \delta\left\|u^{n}\right\|_{L^{2}}+\tau\|f\|_{L^{2}}$. For $w \in V_{N}$ such that $\|w\|_{L^{2}}=R_{0}$ we have $(F(w), w)_{L^{2}}>0$. Then by the Brouwer's type Lemma 1 there exists $w^{*} \in V_{N}$, such that $\left\|w^{*}\right\|_{L^{2}} \leq R_{0}$ and $F\left(w^{*}\right)=0$. Hence $w^{N}=w^{*}-\delta P_{N} u^{n} \in V_{N}$ is solution of (19) and satisfies

$$
\left\|w^{N}\right\|_{L^{2}} \leq 4 \delta\left\|u^{n}\right\|_{L^{2}}+\tau\|f\|_{L^{2}} .
$$

This completes the proof of the Lemma.

\subsection{Passing to the limit}

In order to let $N$ goes to $+\infty$ in (19), we need some compactness argument. To this end, we first state and prove

Lemma 3 The sequence $\left(w^{N}\right)_{N}$ is bounded in $H^{\alpha}$.

Proof : On the one hand, taking $\varphi^{N}=w^{N}+\delta P_{N} u^{n}$ in (19) leads to

$$
\left\|w^{N}\right\|_{L^{2}}^{2} \leq \delta^{2}\left\|P_{N} u^{n}\right\|_{L^{2}}^{2}+\tau\left(f, w^{N}+\delta P_{N} u^{n}\right)_{L^{2}},
$$

that implies

$$
\left\|w^{N}\right\|_{L^{2}} \leq \delta\left\|u^{n}\right\|_{L^{2}}+\tau\|f\|_{L^{2}} .
$$

Then, since $u^{n}$ belongs to $H^{\alpha}$ then the sequence $w^{N}$ is bounded in $L^{2}$. On the other hand, taking $\varphi^{N}=i\left(w^{N}-\delta P_{N} u^{n}\right)$ in (19), we get 


$$
\begin{array}{r}
\left\|\Lambda^{\alpha} w^{N}\right\|_{L^{2}}^{2}=\delta^{2}\left\|\Lambda^{\alpha} P_{N} u^{n}\right\|_{L^{2}}^{2}+\frac{1}{2} \int_{\mathbb{R}}\left(\left|w^{N}\right|^{2}+\left|u^{n}\right|^{2}\right)\left(\left|w^{N}\right|^{2}-\delta^{2}\left|P_{N} u^{n}\right|^{2}\right) d x+ \\
-2 \operatorname{Im} \int_{\mathbb{R}} f \overline{\left(w^{N}-\delta P_{N} u^{n}\right)} d x .
\end{array}
$$

To get an upper bound for the right hand side of (27), we control the main term as follows

$$
\int_{\mathbb{R}}\left(\left|w^{N}\right|^{2}+\left|u^{n}\right|^{2}\right)\left|w^{N}\right|^{2} d x \leq\left(\left\|w^{N}\right\|_{L^{\infty}}^{2}+\left\|u^{n}\right\|_{L^{\infty}}^{2}\right)\left\|w^{N}\right\|_{L^{2}}^{2} \leq K+\frac{1}{8}\left\|\Lambda^{\alpha} w^{N}\right\|_{L^{2}}^{2}
$$

using moreover the interpolation inequality (10), Young inequality and the $L^{2}$ upper bound. This completes the proof of the Lemma.

Proposition 3 There exists at least a solution to (7).

Proof : We now pass to the limit in (19), for $N \geq N_{0}$ and for a given $\varphi^{N_{0}}$ in $V_{N_{0}}$. We know that there exist a subsequence still denoted by $w^{N}$ that converges weakly in $H^{\alpha}$ towards $u^{n+1}$. Passing to the limit in the linear terms of (19) is standard and then omitted. We focus on the nonlinear term, and we aim to prove that

$$
\int_{\mathbb{R}}\left(\left|w^{N}\right|^{2} w^{N}-\left|u^{n+1}\right|^{2} u^{n+1}\right) \bar{\varphi} d x \rightarrow 0 .
$$

We proceed as follows. we consider a smooth cut off function $\rho \in \mathcal{C}_{c}^{\infty}(\mathbb{R})$ such that

$$
\left\{\begin{array}{lll}
\rho(s)=1 & \text { if } & |s| \leq 1 \\
\rho(s)=0 & \text { if } & |s| \geq 2 .
\end{array}\right.
$$

For each $r>0$, let $\rho_{r}(s)=\rho\left(\frac{s}{r}\right)$. On the one hand, up to another subsequence extraction, since $H^{\alpha}(-2 r, 2 r)$ is compactly embedded into $C(-2 r, 2 r)$, we have that

$$
\int_{\mathbb{R}}\left(\left|w^{N}\right|^{2} w^{N}-\left|u^{n+1}\right|^{2} u^{n+1}\right) \rho_{r} \bar{\varphi} d x \rightarrow 0
$$


On the other hand, using the previous $L^{2}$ and $L^{\infty}$ estimates

$$
\int_{\mathbb{R}}\left(\left|w^{N}\right|^{2} w^{N}-\left|u^{n+1}\right|^{2} u^{n+1}\right)\left(1-\rho_{r}\right) \bar{\varphi} d x \leq K\left(\int_{|x| \geq r}|\varphi|^{2} d x\right)^{\frac{1}{2}} .
$$

Then letting first $N \rightarrow+\infty$ and then $r \rightarrow+\infty$ leads to (29). Hence we can pass to the limit for a given $\varphi^{N_{0}}$ in $V^{N_{0}}$. To complete the proof of the Proposition 3 we use that $\bigcup_{N_{0} \geq 0} V^{N_{0}}$ is dense in $H^{\alpha}$ and we conclude by a density argument.

\subsection{Absorbing sets that are positively invariant}

We state and prove

Proposition 4 There exists a bounded set $\mathcal{E} \subset H^{\alpha}$ that is an absorbing set for $S$, and that is positively invariant by $S$.

Proof : We first construct a $L^{2}$ absorbing ball. Set $M_{0}=\frac{2\|f\|_{L^{2}}}{\gamma}$. Consider the $L^{2}$ scalar product of $(7)$ with $u^{n+1}+\delta u^{n}$. We thus obtain

$$
\left\|u^{n+1}\right\|_{L^{2}} \leq \delta\left\|u^{n}\right\|_{L^{2}}+(1-\delta) \frac{\|f\|_{L^{2}}}{\gamma}
$$

Therefore the set $\mathcal{E}_{0}=\left\{u \in H^{\alpha},\|u\|_{L^{2}} \leq M_{0}\right\}$ is positively invariant, and, thanks to the discrete Gronwall Lemma, absorbing for the $L^{2}$ topology; actually

$$
\left\|u^{n}\right\|_{L^{2}} \leq \delta^{n}\left\|u^{0}\right\|_{L^{2}}+\left(1-\delta^{n}\right) \frac{\|f\|_{L^{2}}}{\gamma}
$$

and $u^{n}$ belongs to $\mathcal{E}_{0}$ for $n$ large enough.

We now seek for an absorbing set $\mathcal{E}$ in $H^{\alpha}$ as a susbset of $\mathcal{E}_{0}$. Without loss of generality assume then that $u^{0}$ (and then $u^{n}$ ) belongs to the $L^{2}$ bounded absorbing set. We now follow the same lines as in the continuous case (see [6]). That is, we consider the functional

$$
J(u)=\left\|\Lambda^{\alpha} u\right\|_{L^{2}}^{2}-\frac{1}{2} \int_{\mathbb{R}}|u|^{4} d x+2 \operatorname{Im} \int_{\mathbb{R}} f \bar{u} d x .
$$

Using the Cauchy-Schwarz inequality and bounding by above the nonlinear part of $J(u)$, we get $\forall u \in H^{\alpha}$

$$
J(u) \geq\left\|\Lambda^{\alpha} u\right\|_{L^{2}}^{2}-\frac{1}{2}\|u\|_{L^{\infty}}^{2}\|u\|_{L^{2}}^{2}-2\|f\|_{L^{2}}\|u\|_{L^{2}} .
$$


Then by interpolation inequality (10) and Young inequality, we obtain $\forall u \in \mathcal{E}_{0}$

$$
J(u) \geq \frac{1}{2}\left\|\Lambda^{\alpha} u\right\|_{L^{2}}^{2}-K_{0}
$$

for a fixed $K_{0}=K_{0}(\gamma, f)$.

We seek an equality satisfied by this functional; we then take the scalar product of (7) with $i\left(u^{n+1}-\delta u^{n}\right)$, to get

$$
\begin{aligned}
\left\|\Lambda^{\alpha} u^{n+1}\right\|_{L^{2}}^{2}-\delta^{2}\left\|\Lambda^{\alpha} u^{n}\right\|_{L^{2}}^{2}-\frac{1}{2} \int_{\mathbb{R}}\left(\left|u^{n+1}\right|^{2}+\right. & \left.\left|u^{n}\right|^{2}\right)\left(\left|u^{n+1}\right|^{2}-\delta^{2}\left|u^{n}\right|^{2}\right) d x \\
& =-2 \operatorname{Im} \int_{\mathbb{R}} f \overline{u^{n+1}-\delta u^{n}} d x .
\end{aligned}
$$

This leads to

$$
J\left(u^{n+1}\right)=\delta^{2} J\left(u^{n}\right)+\frac{1}{2}\left(1-\delta^{2}\right) \int_{\mathbb{R}}\left|u^{n}\right|^{2}\left|u^{n+1}\right|^{2} d x+2 \delta(1-\delta) \operatorname{Im} \int_{\mathbb{R}} f \overline{u^{n}} d x .
$$

On the one hand, we infer from Cauchy-Schwarz inequality and (33) that

$$
\operatorname{Im} \int_{\mathbb{R}} f \overline{u^{n}} d x \leq\|f\|_{L^{2}(\mathbb{R})}\left\|u^{n}\right\|_{L^{2}(\mathbb{R})} \leq K,
$$

since $u_{n}$ remains trapped into the $L^{2}$ absorbing ball. On the other hand, using once more the $L^{2}$ upper bound, the interpolation inequality (10) and Young inequality, we have

$$
\int_{\mathbb{R}}\left|u^{n}\right|^{2}\left|u^{n+1}\right|^{2} d x \leq\left\|u^{n}\right\|_{L^{2}}^{2}\left\|u^{n+1}\right\|_{L^{\infty}}^{2} \leq K+\frac{1}{2}\left\|\Lambda^{\alpha} u^{n+1}\right\|_{L^{2}}^{2} .
$$

Gathering (35) to (40), we thus obtain that

$$
\delta J\left(u^{n+1}\right) \leq\left(1-\frac{1}{2}\left(1-\delta^{2}\right)\right) J\left(u^{n+1}\right) \leq \delta^{2} J\left(u^{n}\right)+(1-\delta) K .
$$

Using the discrete Gronwall lemma we then infer that there exists $K_{1}$ that depends on the data $\gamma, f$ such that the set $\mathcal{E}=\left\{u \in \mathcal{E}_{0}, J(u) \leq K_{1}\right\}$ is a bounded absorbing set in $H^{\alpha}$ that is positively invariant by $S$. In the remaining of the paper, we denote by $M_{1}$ the radius of the smallest ball that contains $\mathcal{E}$. 


\subsection{Continuity of the map $S$ on $\mathcal{E}$.}

We prove here that if $\tau$ is small enough depending on $M_{1}$, then the map $S: \mathcal{E} \rightarrow \mathcal{E}$ is one to one and continuous for the $H^{\alpha}$ topology. This completes the proof of Theorem 1.

Lemma 4 There exists a constant $C$ such that if $C \tau M_{1}^{2}<1$. Then the map $S: \mathcal{E} \rightarrow \mathcal{E}$ is a Lipschitz map for the $H^{\alpha}$ topology.

Proof : Set $u^{n+1}=S u^{n}, v^{n+1}=S v^{n}$ with $u^{n}, v^{n} \in \mathcal{E}$. We denote $w^{n}=u^{n}-v^{n}$ the difference between the two discrete trajectories. Then, $w^{n}$ verifies

$$
\begin{array}{r}
\frac{1}{\tau}\left(w^{n+1}-\delta w^{n}\right)-\frac{i}{2}(-\Delta)^{\alpha}\left(w^{n+1}+\delta w^{n}\right)+\frac{i}{4}\left(\left(\left|u^{n+1}\right|^{2}+\left|u^{n}\right|^{2}\right)\left(w^{n+1}+\delta w^{n}\right)+\right. \\
\frac{i}{4}\left(\left|u^{n+1}\right|^{2}-\left|v^{n+1}\right|^{2}+\left|u^{n}\right|^{2}-\left|v^{n}\right|^{2}\right)\left(v^{n+1}+\delta v^{n}\right)=0 .
\end{array}
$$

We first take the scalar product of $(42)$ with $i\left(w^{n+1}-\delta w^{n}\right)$ to obtain,

$$
\begin{gathered}
\| \Lambda^{\alpha} w^{n+1}||_{L^{2}}^{2}-\left.\delta^{2}|| \Lambda^{\alpha} w^{n}\right|_{L^{2}} ^{2}=\frac{1}{2} \int_{\mathbb{R}}\left(\left|u^{n+1}\right|^{2}+\left|u^{n}\right|^{2}\right)\left(\left|w^{n+1}\right|^{2}-\delta^{2}\left|w^{n}\right|^{2}\right) \\
+\frac{1}{2} \operatorname{Re} \int_{\mathbb{R}}\left(\left|u^{n+1}\right|^{2}-\left|v^{n+1}\right|^{2}+\left|u^{n}\right|^{2}-\left|v^{n}\right|^{2}\right)\left(v^{n+1}+\delta v^{n}\right) \overline{\left(w^{n+1}-\delta w^{n}\right)} .
\end{gathered}
$$

Using the fact that $u^{n}, v^{n}$ remains trapped in $\mathcal{E}$ that is a bounded set of $L^{\infty}$, we then obtain straightforwardly

$$
\left\|\Lambda^{\alpha} w^{n+1}\right\|_{L^{2}}^{2}-\delta^{2}\left\|\Lambda^{\alpha} w^{n}\right\|_{L^{2}}^{2} \leq C M_{1}^{2}\left(\left\|w^{n}\right\|_{L^{2}}^{2}+\left\|w^{n+1}\right\|_{L^{2}}^{2}\right)
$$

We now take the scalar product of $(42)$ with $\left(w^{n+1}+\delta w^{n}\right)$ to get

$\left\|w^{n+1}\right\|_{L^{2}}^{2}-\delta^{2}|| w^{n} \|_{L^{2}}^{2}=\frac{\tau}{4} \operatorname{Im} \int_{\mathbb{R}}\left(\left|u^{n+1}\right|^{2}-\left|v^{n+1}\right|^{2}+\left|u^{n}\right|^{2}-\left|v^{n}\right|^{2}\right)\left(v^{n+1}+\delta v^{n}\right) \overline{\left(w^{n+1}+\delta w^{n}\right)}$

and proceeding as for (43), we get

$$
\left\|w^{n+1}\right\|_{L^{2}}^{2}-\delta^{2}\left\|w^{n}\right\|_{L^{2}}^{2} \leq C \tau M_{1}^{2}\left(\left\|w^{n}\right\|_{L^{2}}^{2}+\left\|w^{n+1}\right\|_{L^{2}}^{2}\right) .
$$

We choose $\tau>0$ small enough such that $C \tau M_{1}^{2}<1$ and setting $C_{2}=\frac{\delta^{2}+C_{1} \tau M_{1}^{2}}{1-C \tau M_{1}^{2}}$ then we infer from (46)

$$
\left\|w^{n+1}\right\|_{L^{2}}^{2} \leq C_{2}\left\|w^{n}\right\|_{L^{2}}^{2} .
$$


Using (47) in (44), we deduce that

$$
\left\|w^{n+1}\right\|_{H^{\alpha}} \leq C_{3}\left\|w^{n}\right\|_{H^{\alpha}}
$$

that completes the proof of the Lemma.

\section{$5 \quad$ Proof of Theorems 2 and 3}

In this section, we prove the existence of a compact global attractor in $H^{\alpha}$. The proof relies on a splitting argument first used in [20] in the continuous case, that allows us to apply the asymptotic compactness Theorem (see Theorem I.1.1 [25]). The key proposition reads as follows

Proposition 5 For any $\eta>0$, a trajectory $u^{n}$ splits as $u^{n}=v^{n}+w^{n}$ where, for $n$ large enough, $\left\|w^{n}\right\|_{H^{\alpha}} \leq \eta$, and, for any $n, v^{n}$ is trapped into a bounded set $\mathcal{B}_{\eta}$ of $H^{2 \alpha} \cap L^{2}\left(\mathbb{R},\left(1+x^{2}\right) d x\right)$.

The proof of this Proposition uses the commutator estimate. We then prove that thanks to the famous. J. Ball argument, it turns out that this global attractor is also a compact subset in $H^{\alpha}$. This is standard and omitted for the sake of conciseness.

\subsection{The splitting method}

For any small $\eta$ in $] 0,1]$, we consider a function $f_{\eta} \in \mathcal{S}$, such that,

$$
\left\|f-f_{\eta}\right\|_{L^{2}} \leq \eta
$$

We split $u^{n}$ solution of (7) as $u^{n}=v^{n}+w^{n}$, where $v^{n}$ is the solution to

$$
\begin{gathered}
\frac{1}{\tau}\left(v^{n+1}-\delta v^{n}\right)-\frac{i}{2}(-\Delta)^{\alpha}\left(v^{n+1}+\delta v^{n}\right)+\frac{i}{4}\left(\left|u^{n+1}\right|^{2}+\left|u^{n}\right|^{2}\right)\left(v^{n+1}+\delta v^{n}\right) \\
=f_{\eta}, \\
v^{0}=0 .
\end{gathered}
$$

Then $w^{n}=u^{n}-v^{n}$ is the solution to

$$
\begin{aligned}
& \frac{1}{\tau}\left(w^{n+1}-\delta w^{n}\right)-\frac{i}{2}(-\Delta)^{\alpha}\left(w^{n+1}+\delta w^{n}\right)+\frac{i}{4}\left(\left|u^{n+1}\right|^{2}+\left|u^{n}\right|^{2}\right)\left(w^{n+1}+\delta w^{n}\right) \\
& =f-f_{\eta}
\end{aligned}
$$




$$
w^{0}=u^{0}
$$

Proposition 6 The sequence $\left(v^{n}\right)_{n}$ is well defined and belongs to $H^{2 \alpha}$.

Proof : the equation (50) reads also

$$
\begin{array}{r}
\quad v^{n+1}-\frac{i \tau}{2}(-\Delta)^{\alpha} v^{n+1}+\frac{i \tau}{4}\left(\left|u^{n+1}\right|^{2}+\left|u^{n}\right|^{2}\right) v^{n+1} \\
=\delta\left(v^{n}+\frac{i \tau}{2}(-\Delta)^{\alpha} v^{n}-\frac{i \tau}{4}\left(\left|u^{n+1}\right|^{2}+\left|u^{n}\right|^{2}\right) v^{n}\right)+f_{\eta} .
\end{array}
$$

The operator $I d-\frac{i \tau}{2}(-\Delta)^{\alpha}+\frac{i \tau}{4}\left(\left|u^{n+1}\right|^{2}+\left|u^{n}\right|^{2}\right)$ is invertible and its inverse maps $L^{2}$ into $H^{2 \alpha}$. We then prove recursively on $n$ that $v^{n}$ belongs to $H^{2 \alpha}$.

We now prove that the sequence $w^{n}$ remains asymptotically small.

Lemma 5 There exists $\kappa$ such that for $n$ large enough

$$
\left\|w^{n}\right\|_{H^{\alpha}} \leq \kappa \eta
$$

Proof : we consider the scalar product of (52) with $w^{n+1}+\delta w^{n}$. This yields

$$
\frac{1}{\tau}\left(\left\|w^{n+1}\right\|_{L^{2}}^{2}-\delta^{2}\left\|w^{n}\right\|_{L^{2}}^{2}\right)=\operatorname{Re} \int_{\mathbb{R}}\left(f-f_{\eta}\right) \overline{w^{n+1}+\delta w^{n}} d x .
$$

We use the Cauchy-Schwarz inequality and (49) to obtain

$$
\left\|w^{n+1}\right\|_{L^{2}} \leq \delta\left\|w^{n}\right\|_{L^{2}}+\tau \eta
$$

The discrete Gronwall lemma yields that the sequence $w^{n}$ remains small in $L^{2}$, i.e.

$$
\left\|w^{n}\right\|_{L^{2}} \leq K \eta
$$

We now proceed to the $H^{\alpha}$ estimate. We consider the scalar product of (52) with $i\left(w^{n+1}-\delta w^{n}\right)$ to obtain

$$
\begin{aligned}
\left\|\Lambda^{\alpha} w^{n+1}\right\|_{L^{2}}^{2}= & \delta^{2}\left\|\Lambda^{\alpha} w^{n}\right\|_{L^{2}}^{2}+\frac{1}{2} \int_{\mathbb{R}}\left(\left|u^{n+1}\right|^{2}+\left|u^{n}\right|^{2}\right)\left(\left|w^{n+1}\right|^{2}-\delta^{2}\left|w^{n}\right|^{2}\right) d x \\
& -2 \operatorname{Im} \int_{\mathbb{R}}\left(f-f_{\eta} \overline{\left(w^{n+1}-\delta w^{n}\right)} d x .\right.
\end{aligned}
$$


Using estimate (58) and the fact that $u^{n}$ remains in a bounded set of $L^{\infty}$ we have that

$$
\left\|\Lambda^{\alpha} w^{n+1}\right\|_{L^{2}}^{2} \leq \delta^{2}\left\|\Lambda^{\alpha} w^{n}\right\|_{L^{2}}^{2}+K \eta^{2} .
$$

This completes the proof of Lemma 5, thanks to discrete Gronwall Lemma.

We then prove an estimate on the sequence $v^{n}$

Proposition 7 The sequence $\left(v^{n}\right)$ is uniformly bounded in $H^{2 \alpha} \cap L^{2}(\mathbb{R},(1+$ $\left.x^{2}\right) d x$, that is there exists a constant $K$ such that for all $u^{0} \in \mathcal{E}$ we have, for any $n \geq 0$

$$
\left\|x v^{n}\right\|_{L^{2}}+\tau\left\|v^{n}\right\|_{H^{2 \alpha}} \leq K
$$

Proof : Since $v_{n}=u_{n}-w_{n}$ we already know that $v_{n}$ remains bounded in $H^{\alpha}$. We begin with the bound for $x v^{n}$ in $L^{2}$. Introduce a cutoff function $\varphi$ in $\mathcal{S}$ such that $\varphi$ is compactly supported, $\varphi=1$ on a neighborhood of 0 , and $0 \leq \varphi \leq 1$. Set $V^{n}(x)=x \varphi\left(\frac{x}{N}\right) v^{n}$, and $\varphi_{N}(x)=\varphi\left(\frac{x}{N}\right)$.

Let us observe that equation (50) reads also

$$
\begin{aligned}
& \frac{1}{\tau}\left(V^{n+1}-\delta V^{n}\right)-\frac{i}{2}(-\Delta)^{\alpha}\left(V^{n+1}+\delta V^{n}\right)+\frac{i}{4}\left(\left|u^{n+1}\right|^{2}+\left|u^{n}\right|^{2}\right)\left(V^{n+1}+\delta V^{n}\right) \\
& =x \varphi_{N} f_{\eta}+\frac{i}{2}\left[x \varphi_{N},(-\Delta)^{\alpha}\right]\left(v^{n+1}+\delta v^{n}\right),
\end{aligned}
$$

Now, taking the $L^{2}$ inner product of (62) with $V^{n+1}+\delta V^{n}$ we obtain

$$
\begin{aligned}
& \frac{1}{\tau}\left(\left\|V^{n+1}\right\|_{L^{2}}^{2}-\delta^{2}\left\|V^{n}\right\|_{L^{2}}^{2}\right) \\
& \left.=\operatorname{Re} \int_{\mathbb{R}} x \varphi_{N} f_{\eta} \overline{V^{n+1}+\delta V^{n}} d x+\operatorname{Im} \frac{1}{2} \int_{\mathbb{R}}\left[x \varphi_{N},(-\Delta)^{\alpha}\right)\right]\left(v^{n+1}+\delta v^{n}\right) \overline{V^{n+1}+\delta V^{n}} d x .
\end{aligned}
$$

We are going to estimate the right hand side of (63). First, the first term is bounded by above as follows

$$
\left|\int_{\mathbb{R}} x \varphi_{N} f_{\eta} \overline{V^{n+1}+\delta V^{n}} d x\right| \leq\left\|x \varphi_{N} f_{\eta}\right\|_{2}\left(\left\|V^{n+1}\right\|_{2}+\delta\left\|V^{n}\right\|_{2}\right)
$$


Then, the second term is bounded by above by

$$
\frac{1}{2}\left\|\left[x \varphi_{N},(-\Delta)^{\alpha}\right]\left(v^{n+1}+\delta v^{n}\right)\right\|_{L^{2}}\left(\left\|V^{n+1}\right\|_{2}+\delta\left\|V^{n}\right\|_{2}\right) .
$$

We now use Proposition 1 to get, using also that $v^{n}$ remains bounded in $H^{\alpha}$

$$
\left\|\left[x \varphi_{N},(-\Delta)^{\alpha}\right]\left(v^{n+1}+\delta v^{n}\right)\right\|_{L^{2}} \leq K\left(\left\|\xi \widehat{x \varphi_{N}}\right\|_{L^{1}}+\left\|\xi^{2 \alpha} \widehat{x \varphi_{N}}\right\|_{L^{1}}\right) .
$$

Actually

$$
\left\|\xi \widehat{x \varphi_{N}}\right\|_{L^{1}}=N^{2} \int_{\mathbb{R}}\left|\xi \| \partial_{\xi} \hat{\varphi}(N \xi) d \xi\right| \leq C .
$$

The same computation leads to

$$
\left\|\xi^{2 \alpha} \widehat{x \varphi_{N}}\right\|_{L^{1}} \text { converges to } 0,
$$

when $N \rightarrow+\infty$. Therefore we have, gathering these in (63)

$$
\left\|V^{n+1}\right\|_{L^{2}}-\delta\left\|V^{n}\right\|_{L^{2}} \leq \tau K .
$$

Again thanks to the discrete Gronwall lemma, we deduce that

$$
\left\|x \varphi\left(\frac{x}{N}\right) v^{n+1}\right\|_{L^{2}} \leq K .
$$

Hence, letting $N$ goes to $+\infty$, thanks to the Fatou Lemma we deduce that,

$$
\left\|x v^{n+1}\right\|_{L^{2}} \leq K .
$$

Thus, we deduce that $v^{n}$ is bounded in $H^{\alpha} \cap L^{2}\left(\mathbb{R},\left(1+x^{2}\right) d x\right)$.

We now proceed to the $H^{2 \alpha}$ estimate. Taking the inner product of (50) with $i(-\Delta)^{\alpha}\left(v^{n+1}-\delta v^{n}\right)$, we get

$$
\begin{aligned}
& \frac{1}{2}\left(\left\|(-\Delta)^{\alpha} v^{n+1}\right\|_{L^{2}}^{2}-\delta^{2}\left\|(-\Delta)^{\alpha} v^{n}\right\|_{L^{2}}^{2}\right) \\
& =\frac{1}{4} \operatorname{Re} \int_{\mathbb{R}}\left(\left|u^{n+1}\right|^{2}+\left|u^{n}\right|^{2}\right)\left(v^{n+1}+\delta v^{n}\right)(-\Delta)^{\alpha}\left(v^{n+1}-\delta v^{n}\right) d x \\
& -\operatorname{Im} \int_{\mathbb{R}} f_{\eta}(-\Delta)^{\alpha}\left(v^{n+1}-\delta v^{n}\right) d x .
\end{aligned}
$$


Applying Cauchy-Schwarz inequality and using the fact that the sequences $\left(u^{n}\right)_{n}$ and $\left(v^{n}\right)_{n}$ are bounded in $H^{\alpha}$ we deduce that,

$$
\left\|v^{n+1}\right\|_{H^{2 \alpha}} \leq \delta\left\|v^{n}\right\|_{H^{2 \alpha}}+K .
$$

Thus by the discrete Gronwall Lemma and thanks to $v^{0}=0$ we get,

$$
\left\|v^{n+1}\right\|_{H^{2 \alpha}} \leq \frac{K}{\tau} .
$$

\subsection{Existence of the global Attractor}

We have constructed a splitting $u^{n}=v^{n}+w^{n}$ where $w^{n}$ is small enough in $H^{\alpha}$ and $v^{n}$ belongs to a bounded set of $H^{2 \alpha} \cap L^{2}\left(\mathbb{R},\left(1+x^{2}\right) d x\right) \subset H^{\alpha}$. To establish the proof of compactness of the trajectories in $H^{\alpha}$, we use the following classical compact embedding.

Lemma 6 The embedding $H^{2 \alpha} \cap L^{2}\left(\mathbb{R},\left(1+x^{2}\right) d x\right) \hookrightarrow H^{\alpha}$ is compact.

For any $\eta$, any trajectory $u^{n}$ is trapped into the sum of a compact set $\mathcal{B}_{\eta}$ in $H^{\alpha}$ (since $\mathcal{B}_{\eta}$ is bounded in $H^{2 \alpha} \cap L^{2}\left(\mathbb{R},\left(1+x^{2}\right) d x\right)$ ) and a ball of radius $\eta$ centered at 0 . Then the trajectory is covered by a finite sum of balls or radii $2 \eta$ that ensures the asymptotic compactness of the trajectory. Now, the assumptions of Theorem I.1.1 [25] are satisfied and we have a compact global attractor $\mathcal{A}_{\tau}$ in $H^{\alpha}$ for $S$.

\subsection{Dimension of the attractor}

We prove that the global attractor has finite fractal dimension. For this purpose, we rely on a result given in [7].

Proposition 8 Let $X$ be a separable Hilbert space and $M$ is a bounded closed set in $X$. Assume that there exists a mapping $S: M \rightarrow X$ such that $M \subseteq S M$ and

1. $S$ is Lipschitz on $M$, i.e., there exists $L_{0}>0$ such that,

$$
\left\|S v_{1}-S v_{2}\right\|_{X} \leq L_{0}\left\|v_{1}-v_{2}\right\|_{X}, \quad v_{1}, v_{2} \in M .
$$


2. For $\|\cdot\|_{Y}$ that is compact seminorm with respect to $\|\cdot\|_{X}$ (i.e $X \subset Y$ is compact) such that

$\left\|S v_{1}-S v_{2}\right\|_{X} \leq \mu\left\|v_{1}-v_{2}\right\|_{X}+K\left(\left\|v_{1}-v_{2}\right\|_{Y}+\left\|S v_{1}-S v_{2}\right\|_{Y}\right), \quad v_{1}, v_{2} \in M ;$

where $0<\mu<1$ and $K>0$ are constants.

Then $M$ is a compact set in $X$ with finite fractal dimension.

To begin with, we prove that if the external force is in $L^{2}\left(\mathbb{R},\left(1+x^{2}\right) d x\right)$, then the global attractor is also included in this space.

Proposition 9 Let $f \in L^{2}\left(\mathbb{R},\left(1+x^{2}\right) d x\right)$. Then the global attractor $\mathcal{A}_{\tau}$ is a bounded set in $H^{\alpha} \cap L^{2}\left(\mathbb{R},\left(1+x^{2}\right) d x\right)$.

Proof: Consider $\left(u^{n}\right)_{n}$ be a global trajectory that belongs the global attractor $\mathcal{A}_{\tau}$. Set $U^{n}(x)=x \varphi\left(\frac{x}{N}\right) u^{n}$ and $\varphi_{N}(x)=\varphi\left(\frac{x}{N}\right)$ as above. Let us observe that equation (7) reads also

$$
\begin{aligned}
& \frac{1}{\tau}\left(U^{n+1}-\delta U^{n}\right)-\frac{i}{2}(-\Delta)^{\alpha}\left(U^{n+1}+\delta U^{n}\right)+\frac{i}{4}\left(\left|u^{n+1}\right|^{2}+\left|u^{n}\right|^{2}\right)\left(U^{n+1}+\delta U^{n}\right) \\
& =x \varphi_{N} f+\frac{i}{2}\left[x \varphi_{N},(-\Delta)^{\alpha}\right]\left(u^{n+1}+\delta u^{n}\right) .
\end{aligned}
$$

Now, taking the $L^{2}$ inner product of (74) with $U^{n+1}+\delta U^{n}$ we obtain

$$
\begin{aligned}
& \frac{1}{\tau}\left(\left\|U^{n+1}\right\|_{L^{2}}^{2}-\delta^{2}\left\|U^{n}\right\|_{L^{2}}^{2}\right) \\
& =\operatorname{Re} \int_{\mathbb{R}} x \varphi_{N} f \overline{U^{n+1}+\delta U^{n}} d x+\operatorname{Im} \frac{1}{2} \int_{\mathbb{R}}\left[x \varphi_{N},(-\Delta)^{\alpha}\right]\left(u^{n+1}+\delta u^{n}\right) \overline{U^{n+1}+\delta U^{n}} d x .
\end{aligned}
$$

We are going to estimate the right hand side of (75). The first term is bounded by above as follows

$$
\left|\int_{\mathbb{R}} x \varphi_{N} f \overline{U^{n+1}+\delta U^{n}} d x\right| \leq\left\|x \varphi_{N} f\right\|_{2}\left(\left\|U^{n+1}\right\|_{2}+\delta\left\|U^{n}\right\|_{2}\right) .
$$

and the second term is bounded by above by

$$
\frac{1}{2}\left\|\left[x \varphi_{N},(-\Delta)^{\alpha}\right]\left(u^{n+1}+\delta u^{n}\right)\right\|_{L^{2}}\left(\left\|U^{n+1}\right\|_{2}+\delta\left\|U^{n}\right\|_{2}\right) .
$$


We now use Proposition 1 to get, using also that $u^{n}$ remains bounded in $H^{\alpha}$

$$
\left\|\left[x \varphi_{N},(-\Delta)^{\alpha}\right]\left(u^{n+1}+\delta u^{n}\right)\right\|_{L^{2}} \leq K\left(\left\|\xi \widehat{x \varphi_{N}}\right\|_{L^{1}}+\left\|\xi^{2 \alpha} \widehat{x \varphi_{N}}\right\|_{L^{1}}\right) .
$$

Proceeding as in (67) and (68), we know that the right hand side of (78) is bounded independently of $N$. Therefore

$$
\left\|U^{n+1}\right\|_{L^{2}} \leq \delta\left\|U^{n}\right\|_{L^{2}}+\tau K .
$$

Using the discrete Gronwall lemma we then have

$$
\left\|U^{0}\right\|_{L^{2}} \leq \delta^{n}|| U^{-n} \|_{L^{2}}+K
$$

Letting $n \rightarrow+\infty$ leads to

$$
\left\|U^{0}\right\|_{L^{2}} \leq K
$$

Letting $N \rightarrow+\infty$ completes the proof of the proposition.

We now complete the proof of Theorem 3. We know that the attractor is included in $H^{\alpha} \cap L^{2}\left(\mathbb{R},\left(1+x^{2}\right) d x\right)$. We plan to apply Proposition 8 with $X=$ $H^{\alpha} \cap L^{2}\left(\mathbb{R},\left(1+x^{2}\right) d x\right), Y=L^{\infty} \cap H^{2 \alpha-1}$ and $M=\mathcal{A}_{\tau}$. Due to (48), we know that $S$ is a Lipschitz map for the $H^{\alpha}$ norm; to check the first assumption it remains to prove the same property for the weighted $L^{2}$ norm. We proceed as follows; consider $u^{n}, v^{n} \in \mathcal{A}_{\tau}$ and set $u^{n+1}=S u^{n}, v^{n+1}=S v^{n}$. Consider also $U^{n}(x)=x \varphi\left(\frac{x}{N}\right) u^{n}$ and $V^{n}(x)=x \varphi\left(\frac{x}{N}\right) v^{n}$, where $\varphi_{N}(x)=\varphi\left(\frac{x}{N}\right)$ is as above. Set $W^{n}=U^{n}-V^{n}$ and $w^{n}=u^{n}-v^{n}$. Hence, we get

$$
\begin{aligned}
& \frac{1}{\tau}\left(W^{n+1}-\delta W^{n}\right)-\frac{i}{2}(-\Delta)^{\alpha}\left(W^{n+1}+\delta W^{n}\right)+\frac{i}{4}\left(\left(\left|u^{n+1}\right|^{2}+\left|u^{n}\right|^{2}\right)\left(W^{n+1}+\delta W^{n}\right)+\right. \\
& \frac{i}{4}\left(\left|u^{n+1}\right|^{2}-\left|v^{n+1}\right|^{2}+\left|u^{n}\right|^{2}-\left|v^{n}\right|^{2}\right)\left(V^{n+1}+\delta V^{n}\right)=\frac{i}{2}\left[x, \varphi_{N}(-\Delta)^{\alpha}\right]\left(w^{n+1}+\delta w^{n}\right) .
\end{aligned}
$$

Now, taking the $L^{2}$ inner product of (82) with $W^{n+1}+\delta W^{n}$ we obtain

$$
\begin{aligned}
& \frac{1}{\tau}\left(\left\|W^{n+1}\right\|_{L^{2}}^{2}-\delta^{2}\left\|W^{n}\right\|_{L^{2}}^{2}\right) \\
& =\frac{1}{4} \operatorname{Im} \int_{\mathbb{R}}\left[\left(\left|u^{n+1}\right|^{2}-\left|v^{n+1}\right|^{2}\right)+\left(\left|u^{n}\right|^{2}-\left|v^{n}\right|^{2}\right)\right]\left(V^{n+1}+\delta V^{n}\right) \overline{\left(W^{n+1}+\delta W^{n}\right)} \\
& -\frac{1}{2} \operatorname{Im} \int_{\mathbb{R}}\left[x \varphi_{N},(-\Delta)^{\alpha}\right]\left(w^{n+1}+\delta w^{n}\right) \overline{W^{n+1}+\delta W^{n}} d x .
\end{aligned}
$$


Using that the global attractor is bounded in $H^{\alpha} \cap L^{2}\left(\mathbb{R},\left(1+x^{2}\right) d x\right)$ we then obtain, for some constant $K$

$$
\begin{aligned}
& \frac{1}{\tau}\left(\left\|W^{n+1}\right\|_{L^{2}}-\delta\left\|W^{n}\right\|_{L^{2}}\right) \\
& \leq K\left(\left\|w^{n+1}\right\|_{L^{\infty}}+\left\|w^{n}\right\|_{L^{\infty}}+\left\|\left[x \varphi_{N},(-\Delta)^{\alpha}\right]\left(w^{n+1}+\delta w^{n}\right)\right\|_{L^{2}}\right) .
\end{aligned}
$$

A straightforward application of Proposition 1, using also (67) and (68), leads to

$$
\begin{aligned}
& \frac{1}{\tau}\left(\left\|W^{n+1}\right\|_{L^{2}}-\delta\left\|W^{n}\right\|_{L^{2}}\right) \\
& \leq K\left(\left\|w^{n+1}\right\|_{L^{\infty}}+\left\|w^{n}\right\|_{L^{\infty}}+\left\|w^{n+1}\right\|_{H^{2 \alpha-1}}+\left\|w^{n}\right\|_{H^{2 \alpha-1}}\right) .
\end{aligned}
$$

We now let $N \rightarrow+\infty$ in (85). Using that $H^{\alpha} \subset L^{\infty} \cap H^{2 \alpha-1}$ and the fact that $S: w^{n} \mapsto w^{n+1}$ is Lipschitz on $H^{\alpha}$ we then infer that the map $S$ is Lipschitz on $H^{\alpha} \cap L^{2}\left(\mathbb{R},\left(1+x^{2}\right) d x\right)$.

We now proceed to the second assumption in Proposition 8. On the one hand, considering the equation satisfied by the difference $w^{n}=u^{n+1}-v^{n+1}$ of two solutions $u^{n+1}=S u^{n}$ and $v^{n+1}=S v^{n}$, we have, adding (44) to (46)

$$
\left\|w^{n+1}\right\|_{H^{\alpha}}^{2} \leq \delta^{2}\left\|w^{n}\right\|_{H^{\alpha}}^{2}+K\left(\left\|w^{n+1}\right\|_{L^{2}}^{2}+\left\|w^{n}\right\|_{L^{2}}^{2}\right) .
$$

On the other hand, it remains to prove the same assumption for the weighted norm. Letting $N \rightarrow+\infty$ in (85) lead to

$$
\begin{array}{r}
\left\|x w^{n+1}\right\|_{L^{2}} \leq \delta\left\|x w^{n}\right\|_{L^{2}}+ \\
K\left(\left\|w^{n+1}\right\|_{L^{\infty}}+\left\|w^{n}\right\|_{L^{\infty}}+\left\|w^{n+1}\right\|_{H^{2 \alpha-1}}+\left\|w^{n}\right\|_{H^{2 \alpha-1}}\right) .
\end{array}
$$

Thanks to (86) and (87), we deduce :

$$
\begin{array}{r}
\left\|w^{n+1}\right\|_{X} \leq \delta\left\|w^{n}\right\|_{X}+ \\
K\left(\left\|w^{n+1}\right\|_{L^{\infty}}+\left\|w^{n}\right\|_{L^{\infty}}+\left\|w^{n+1}\right\|_{H^{2 \alpha-1}}+\left\|w^{n}\right\|_{H^{2 \alpha-1}}+\left\|w^{n+1}\right\|_{L^{2}}+\left\|w^{n}\right\|_{L^{2}}\right) .
\end{array}
$$

Since the embedding $X \subset L^{\infty} \cap H^{2 \alpha-1}$ is compact, this completes the proof of Theorem 3. 
Remark 3 It is worth to point out that our upper bound for the dimension of the attractor depends on $\tau$ and blows up as $\tau$ converges to 0 . This is not satisfactory since the dimension of the continuous limit equation is finite. To our knowledge, to prove an upper bound that is independent of $\tau$ is a difficult open issue.

\section{ACKNOWLEDGEMENTS}

The first author was supported in part by the Labex CEMPI (ANR-11-LABX0007-01). The two last authors acknowledge the financial support of PHC Utique ASEO.

\section{References}

[1] M. Abounouh, H. Al Moatassime, J-P. Chehab, S. Dumont, O. Goubet, Discrete Schrödinger Equations and dissipative dynamical systems, Comm. in Pure and Applied Analysis. 7 (2008), 211-227.

[2] N. Akroune, Regularity of the attractor for a weakly damped nonlinear Schrödinger equation on $\mathbb{R}$, Appl. Math. Lett, 12 (1999), 45-48.

[3] C. Besse, A relaxation scheme for Nonlinear Schrödinger Equations, SIAM J. Num. Anal., 42 (2004), 934-952.

[4] C. Besse, B. Bidegaray and S. Descombes, Order estimates in time of splitting methods for the Nonlinear Schrödinger Equations, SIAM J. Num. Anal., 40 (2002), 26-40.

[5] F. E. Browder, Existence and uniqueness theorems for solutions of nonlinear boundary value problems, Applications of Partial Differential Equations (R. Finn, ed.), American Mathematical Society, Providence, pp. 24-29, 1965. MR 33:6092.

[6] M. Cheng, The attractor of the dissipative coupled fractional Schrödinger equations. Math. Meth. In The Appl. Scie., 37 (2014), 645-656.

[7] I. Chueshov and I. Lasiecka, Attractors for Second-Order Evolution Equations with a Nonlinear Damping. J. of Dyn. and Diff. Equ., 16 (2004), 469-512. 
[8] M. Delfour, M. Fortin and G. Payre, Finite-difference solutions of a nonlinear Schrödinger equation, J. Comput. Phys., 44(2) (1981), 277-288.

[9] E. Ezzoug, O. Goubet and E. Zahrouni, Semi-discrete weakly damped nonlinear 2-D Schrödinger equation, Diff. Int. Equ., 23 (2010), 232-252

[10] O. Goubet, Regularity of the attractor for the weakly damped nonlinear Schrödinger equations, Applicable Anal., 60 (1996), 99-119.

[11] O. Goubet, Asymptotical smoothing effect for nonlinear Schrödinger equation on the two dimensional torus, J. of Diff. Eq., 161(1) (2000), 96-122.

[12] O. Goubet, Regularity of the attractor for a weakly damped nonlinear Schrödinger equation in $\mathbb{R}^{2}$, Ad. in Diff. Equa., 3 (1998), 337-360.

[13] O. Goubet and M. Hussein, Dynamical properties for a relaxation scheme applied to a weakly damped non local nonlinear Schrodinger equation Anal. Sti. ale Univ. Ovidius Const. 17 (2009), 71-82.

[14] O. Goubet and E. Zahrouni, On a time discretization of a weakly damped forced nonlinear Schrödinger equation, Comm. in Pure and Applied Analysis, 7 (2008), 1429-1442.

[15] B. Guo and Z. Huo, Global Well-Posedness for the Fractional Nonlinear Schrödinger Equation, Comm. in Part.Diff. Equ., 36 (2011), 247-255.

[16] J. Hale, Asymptotic behavior of Dissipative Systems, Math. surveys and Monographs, 25 (1988), AMS, Providence.

[17] Y. Hong and Y. Sire, On Fractional Schrödinger Equations in Sobolev Spaces, Comm. on Pure and App. Anal., 14 (2015), 2265-2282.

[18] N. Laskin, Fractional quantum mechanics and Lévy path integrals, Physics Letters A, (2000), 268-298.

[19] N. Laskin, Fractional Schrödinger equation, Physical Review E, 66, (1995), 357-369. 
[20] P. Laurençot, Long time Behaviour for weakly damped driven nonlinear Schrödinger equation in $\mathbb{R}^{N}, N \leq 3$, Nonlinear Differential Equations and Applications, 2 (1995), 357-369.

[21] A. Miranville and S. Zelik, Attractors for dissipative partial differential equations in bounded and unbounded domains, Handbook of Differential Equations, Evolutionary Partial Differential Equations, C.M. Dafermos and M. Pokorny eds., Elsevier, Amsterdam.

[22] G. Raugel, Global attractors in partial differential equations. Handbook of dynamical systems, Vol. 2, 885-982, North-Holland, Amsterdam, 2002.

[23] J.M. Sanz-Serna and J.G. Verwer, Conservative and nonconservative schemes for the solution of the nonlinear Schrödinger equation. IMA J. of Num. Anal., 6 (1986), 25-42.

[24] W. Strauss and L. Vasquez, Numerical solution of a nonlinear Klein-Gordon equation. J. Comput. Phys., 28 (1978), 271-278.

[25] R. Temam, Infinite Dimensional Dynamical Systems in Mechanics and Physics, Springer-Verlag, Second Edition, 1997.

[26] R. Temam, Navier Stokes Equations, North Holland, 1983.

[27] Y. Yan, Attractors and dimensions for discretizations of a weakly damped Schrödinger equations and a sine-Gordon equation, Nonlinear Anal., 20 (1993), 1417-1452.

[28] X. Wang, An Energy Equation for Weakly Damped Driven Nonlinear Schrödinger Equations and Its Application to Their Attractors, Physica D, 88 (1995), 167-175. 